\title{
The Effects of Monobromobimane on Calcium and Phenylarsineoxide- Induced Mitochondrial Swelling and Cytochrome c Release in Isolated Brain Mitochondria
}

\author{
Tsutomu Abe, Norio Takagi, Midori Nakano, Kouichi Tanonaka, and Satoshi Takeo* \\ Department of Pharmacology, Tokyo University of Pharmacy and Life Science; 1432-1 Horinouchi, Hachioji, Tokyo \\ 192-0392, Japan. Received December 10, 2003; accepted January 9, 2004
}

\begin{abstract}
A possible involvement of inhibitory effects of monobromobimane (MBM), a thiol reagent, on the swelling and the release of cytochrome $\mathrm{c}$ in the isolated brain mitochondria was examined. MBM dose-dependently inhibited the calcium and phenylarsineoxide-induced mitochondrial swelling and cytochrome c release. Significant relationships between mitochondrial swelling and cytochrome c release were detected. Furthermore, effects of in vivo treatment with $\mathrm{MBM}$ on neuronal cell damage after transient $(15 \mathrm{~min})$ global ischemia in rats were examined. Infusion of MBM (1 or $3 \mu \mathrm{g} /$ animal) to cerebral ventricles attenuated an increased number of TUNEL-positive cells and neuronal cell death in the hippocampal CA1 region at $72 \mathrm{~h}$ of reperfusion. These results suggest that MBM may have an ability to inhibit mitochondria-associated apoptotic pathways through attenuation of the mitochondrial swelling and the release of cytochrome c.
\end{abstract}

Key words cerebral ischemia; mitochondria; monobromobimane; mitochondrial swelling; cytochrome c

Recent evidence suggests that the mitochondrial permeability transition (MPT) may be associated with damage to cells and subsequent cell death. ${ }^{1-5)}$ Opening of the MPT pore, which occurred in the presence of excessive calcium, ${ }^{2,6)}$ causes uncoupling of oxidative phosphorylation, and also induces mitochondrial swelling. ${ }^{7-9)}$ In addition, oxidative damage to mitochondria in concert with mitochondrial calcium overload causes opening of the MPT pore. ${ }^{10,11)}$ It has been suggested that the mitochondrial swelling results in the release of mitochondrial intermembrane proteins including cytochrome $\mathrm{c}$ and the enhancement of apoptotic process. Conditions of calcium influx into the cell and oxidative damages are frequently observed in ischemia followed by reperfusion. Transient global cerebral ischemia induces a neuronal death in vulnerable brain regions such as the hippocampal CA1 region. ${ }^{12)}$ Several lines of evidence suggest a contribution of apoptotic processes to cell death after transient global cerebral ischemia. ${ }^{13)}$ Therefore, opening of the MPT pore and/or mitochondrial swelling may play a pivotal role in transient ischemia-induced cell death.

Monochlorobimane (MCB) and monobromobimane (MBM) are agents that can bind to sulfydryl groups derived from cysteine residue of low molecular weight thiols, such as glutathione (GSH). Adenine nucleotide translocator (ANT) in the MPT pore has vicinal thiols derived from cysteine, which are considered to alter opening of the MPT pore. ${ }^{14,15)}$ Thus, we have hypothesized that thiol agents may have the ability to prevent neuronal cell death after cerebral ischemia through an inhibition of opening of the MPT pore and/or mitochondrial swelling. To investigate this hypothesis, we have examined the effects of MCB and MBM on $\mathrm{Ca}^{2+}$ and phenylarsineoxide ( $\mathrm{PhAsO}$ )-induced swelling and cytochrome $\mathrm{c}$ release using the isolated brain mitochondria. Furthermore, we conducted histological analysis to examine whether in vivo treatment with MBM might protect neuronal cell damage in the hippocampal CA1 region after cerebral ischemia.

\section{MATERIALS AND METHODS}

Animals All procedures using animals were approved by the Guideline of Experimental Animal Care issued by the Prime Minister's Office of Japan. The protocol of the present study was approved by the Committee of Animal Use and Welfare of Tokyo University of Pharmacy \& Life Science. Male Wistar rats (Charles River Japan Inc., Atsugi, Japan), weighing $250-300 \mathrm{~g}$, were given free access to food and water and conditioned at $23 \pm 1{ }^{\circ} \mathrm{C}$ with a constant humidity of $55 \pm 5 \%$ and a cycle of 12 -h light/12-h darkness.

Preparation of Isolated Mitochondria Isolated mitochondria were prepared according to the method of Sims ${ }^{16)}$ with slight modifications. The forebrain was homogenized $(10 \% \mathrm{wt} / \mathrm{vol})$ in isolation buffer containing $320 \mathrm{~mm}$ sucrose, $10 \mathrm{~mm}$ Tris- $\mathrm{HCl}$ (pH 7.4), 1 mm EDTA, $0.25 \%$ fatty acid-free bovine serum albumin. The homogenate was mixed with an equal volume of $24 \%$ ( $\mathrm{vol} / \mathrm{vol})$ percoll and then layered on a percoll gradient, 40 and $26 \%$, and centrifuged at $30700 \times \boldsymbol{g}$ for $10 \mathrm{~min}$ at $4{ }^{\circ} \mathrm{C}$, yielding a dense "fraction 3 ," followed by two washing steps at $16700 \times \boldsymbol{g}$, and $7300 \times \boldsymbol{g}$ for $10 \mathrm{~min}$ at $4^{\circ} \mathrm{C}$. A final mitochondria pellet was resuspended in isolation buffer for assay and stored on ice.

Measurements of the Swelling Rate of the Isolated Mitochondria The isolated mitochondria was incubated for $5 \mathrm{~min}$ with buffer containing $150 \mathrm{~mm} \mathrm{KCl,} 20 \mathrm{~mm}$ MOPS, $10 \mathrm{~mm}$ Tris $/ \mathrm{HCl}, \mathrm{pH} 7.0,2 \mathrm{~mm}$ nitrirotriacetic acid, $0.5 \mu \mathrm{M}$ rotenone, $0.5 \mu \mathrm{m}$ antimycin $\mathrm{A}$, and $2 \mu \mathrm{M}$ A23187, a calcium ionophore, to ensure complete equilibration of calcium ions across the mitochondrial membrane under de-energized conditions. ${ }^{17)}$ Measurements of the $\mathrm{Ca}^{2+}$ and PhAsO-induced mitochondrial swelling were performed with a spectrophotometer (Ubest-30, JASCO, Tokyo) by measuring the decrease in light scattering at $540 \mathrm{~nm}$ at $30^{\circ} \mathrm{C}$. The swelling rate was calculated from the linear change in the light scattering during $5 \mathrm{~min}$ following the addition of $250 \mu \mathrm{M}$ calcium chloride and $30 \mu \mathrm{M} \mathrm{PhAsO}$.

Western Immunoblot Analysis Amounts of cytochrome $\mathrm{c}$ and cytochrome $\mathrm{c}$ oxidase subunit IV (COX IV) were de- 
termined using samples of the supernatant fluid and pellet, which were obtained by centrifuging the mitochondrial suspension after measuring the mitochondrial swelling. The sample was solubilized by boiling for $5 \mathrm{~min}$ with $62.5 \mathrm{~mm}$ Tris- $\mathrm{HCl}(\mathrm{pH}$ 6.8), 2\% SDS, 5\% $\beta$-mercaptoethanol, 10\% glycerol, and $0.001 \%$ bromophenol blue. Following electrophoresis, the proteins were transferred to a polyvinylidene difluoride membrane (Immobilion PVDF, Millipore, Bedford, MA, U.S.A.). Protein blots were incubated overnight at $4{ }^{\circ} \mathrm{C}$ with anti-cytochrome c antibody (Pharmingen) or anti-COX IV antibody (Molecular Probes), and the bound antibody was detected by enhanced chemiluminescence (Amersham Biosciences Inc., Buckinghamshire, U.K.). Quantification of the immunoreactive band was performed by densitometric scanning of autoradiograms and an image analyzer (ATTO Co., Tokyo, Japan). Care was taken to ensure that bands to be semiquantified were in the linear range of response.

Animal Model of Transient Global Ischemia Transient global ischemia (15 min) was induced by a modified method of Sugio, ${ }^{18)}$ as described by Takagi et al. ${ }^{19)}$ Rats were anesthetized with sodium pentobarbital $(50 \mathrm{mg} / \mathrm{kg}$ i.p.). The right and left second cervical vertebras were exposed, and both visible vertebral arteries were permanently electrocauterized. Twenty-four hours after the electrocauterization, anesthesia was induced with $3 \%$ enflurane and maintained with $1.5 \%$ enflurane in a mixture of oxygen/nitrous oxide $(25 / 75 \%)$. Both common carotid arteries were exposed and occluded with aneurysm clips for $15 \mathrm{~min}$, at which the clips were removed and the rat was allowed to recover. Rectal temperature was monitored throughout surgery, and maintained at 37.0 $37.5^{\circ} \mathrm{C}$ using a heating pad and a heating lamp. The present experiments were performed only if animals showed a completely flat bitemporal electroencephalogram during duration of the carotid artery occlusion. Sham-operated animals received exactly the same surgical procedures without arterial occlusion.

In Vivo MBM Administration To examine the potential protective effect of MBM against ischemic cell death, MBM was administered into the right cerebral ventricle. Rats were anesthetized with pentobarbital and placed in a stereotaxic apparatus. After making a burr hole at the parietal skull $0.8 \mathrm{~mm}$ posterior and $1.5 \mathrm{~mm}$ lateral from bregma, 22-gauge needles were inserted into the right cerebral ventricle at a depth of $4.0 \mathrm{~mm}$ from the cortical surface. Thirty minutes before ischemia, each animal received a single ventricular infusion of $1-3 \mu \mathrm{g} \mathrm{MBM} / 5 \mu \mathrm{l}$ over a 3 -min time period. Infusion of the same volume of saline served as control for MBM treatment. Rectal temperature was monitored throughout injection of drug, and maintained at $37.0-37.5^{\circ} \mathrm{C}$ using a heating pad and a heating lamp.

Histological Analysis Transient global ischemia-induced neuronal damage was assessed by histological analysis of brain sections at the level of the hippocampus at $72 \mathrm{~h}$ of reperfusion. Coronal sections $(7 \mu \mathrm{m})$ were cut with microtome and stained with cresyl violet acetate or terminal deoxynucleotidyl transferase-mediated dUTP-biotin in situ nick end labeling (TUNEL). The number of pyramidal cells or TUNEL-positive cells in the hippocampal CA1 was counted in five to seven sections from an animal. Results were expressed as the average number of cells per $\mathrm{mm}^{2}$ of the hippocampal CA1 region. The microscopic observations were performed by a person unaware of the study group.

Statistics The results are expressed as means \pm S.E.M. Statistical comparison among multiple groups was evaluated by ANOVA followed by post-hoc Dunnett's test. The relationship between two parameters was calculated by the least squares method. Differences with a probability of less than $5 \%$ were considered to be significant $(p<0.05)$.

\section{RESULTS}

The Effect of MCB and MBM on the Swelling Rate in Isolated Brain Mitochondria in Vitro To determine mitochondrial swelling rate and cytochrome c release, we used $\mathrm{Ca}^{2+}$ and $\mathrm{PhAsO}$ as inducers. Upon addition of the inducers, the swelling rate increased compared with vehicle-treated group (Fig. 1a). This mitochondrial swelling induced a release of cytochrome c (cyt. c), but not cytochrome c oxidase subunit IV (COX IV), from mitochondria (Fig. 1b). We next examined the effect of thiol reagents, MCB and MBM, on $\mathrm{Ca}^{2+}$ and $\mathrm{PhAsO}$-induced mitochondrial swelling in vitro. The mitochondrial swelling rate was decreased with increased concentrations of MCB. MCB at the concentration of $1 \mathrm{~mm}$ significantly decreased the swelling rate $(n=6$ each, Fig. 2a). Treatment with MBM at the concentrations ranging from 30 to $300 \mu \mathrm{M}$ significantly attenuated the swelling rate in a concentration-dependent manner ( $n=6$ each, Fig. $2 b)$. We have confirmed that MCB and MBM per se did not change the values of absorbance.

The Effect of MBM on the Cytochrome c Release from Isolated Brain Mitochondria in Vitro As the ability of MBM to inhibit mitochondrial swelling was higher than that of MCB (Fig. 2), we next focused on the effect of MBM on the release of cytochrome $\mathrm{c}$ from the isolated brain mitochondria in vitro. Figure 3 a shows that treatment with MBM significantly attenuated the $\mathrm{Ca}^{2+}$ and $\mathrm{PhAsO}$-induced cytochrome $\mathrm{c}$ release in a concentration-dependent manner. Furthermore, there was the close relation between mitochondrial swelling and release of cytochrome c (Fig. 3b).

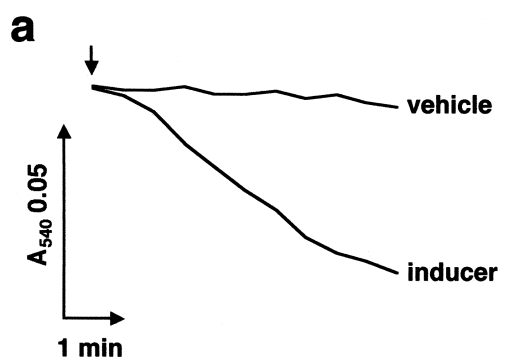

b

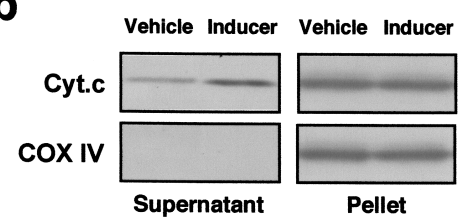

Fig. 1.

(a) Mitochondrial swelling was induced by $250 \mu \mathrm{mol} / 1$ calcium chloride and $30 \mu \mathrm{mol} / 1 \mathrm{PhAsO}$ (inducer) and measured spectrophotometrically as a decrease in A540. (b) After the measurement of swelling, mitochondrial suspension was centrifuged. The supernatant fluid and mitochondrial pellets were used to detect cytochrome $\mathrm{c}$ or COX IV by Western blotting. $\mathrm{Ca}^{2+}$ and $\mathrm{PhAsO}$ selectively induced the release of cytochrome c (cyt. c), but not COX IV. 

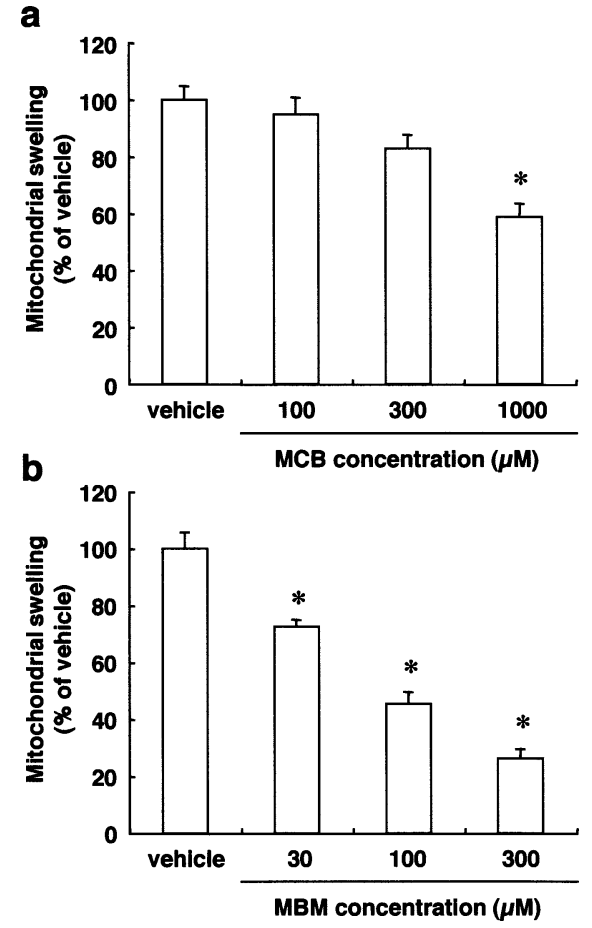

Fig. 2. Effects of MCB (a) or MBM (b) on Mitochondrial Swelling in Vitro

Treatment with MCB or MBM was performed $5 \mathrm{~min}$ before the addition of the inducer. Values represent mean percentages of vehicle treatment \pm S.E.M. $(n=6$ each). $*$ Significantly different from the value for vehicle treatment $(p<0.05)$.

The Effect of MBM on Neuronal Cell Death in the Hippocampal CA1 Region at $72 \mathrm{~h}$ after Transient Cerebral Ischemia To elucidate the ability of MBM to prevent ischemic injury in vivo, we examined the effect of MBM on neuronal cell death in the CA1 region after transient global ischemia. Treatment with MBM dose-dependently attenuated the increased number of TUNEL-positive cells (Figs. $4 \mathrm{a}-\mathrm{d}$, Table 1) and neuronal cell death (Figs. 4e-h, Table 1) in the hippocampal CA1 region at $72 \mathrm{~h}$ after transient cerebral ischemia (Fig. 4, Table 1).

\section{DISCUSSION}

In the present study, we at first determined whether MCB and MBM have the ability to inhibit mitochondrial swelling in vitro. Both agents significantly inhibited the mitochondrial swelling, suggesting the possibility of the inhibitory effects of MCB and MBM on the MPT pore.

An adenine nucleotide translocator (ANT), which is a component of the MPT pore complex, ${ }^{20}$ ) has vicinal thiols derived from cysteine. Furthermore, the thiol cross-linking of ANT has been shown to cause conformational changes in the MPT pore and then open the pore in the isolated mitochondria. ${ }^{14,21)} \mathrm{PhAsO}$ used in the present study was reported to induce or facilitate calcium-induced opening of the MPT pore in the liver, heart, and brain., ${ }^{4,21-23)}$ Therefore, thiol reagents $\mathrm{MCB}$ and MBM are capable of preventing the formation of thiol cross-linking of ANT, consequently reduces the opening of the MPT pore and/or mitochondrial swelling in vitro.

We demonstrated that the effect of MBM was stronger than that of MCB with respect to the potency to inhibit mitochondrial swelling, although further studies will be needed to
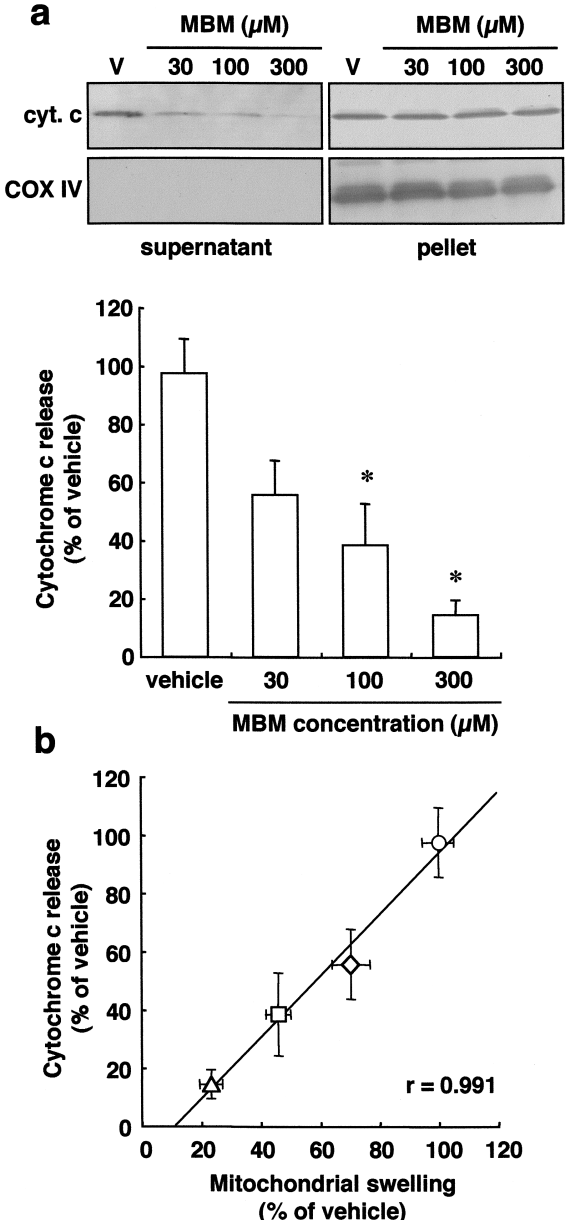

Fig. 3. (a) Effects of MBM on the Release of Cytochrome c from the Mitochondria

The supernatant fluid and mitochondrial pellets were used to detect cytochrome c (cyt. c) or COX IV by Western blotting (upper panel). Bands corresponding to cyt. $\mathrm{c}$ in the supernatant were scanned (lower panel). Values represent mean percentages of vehicle treatment \pm S.E.M. $(n=4$ each $)$. $*$ Significantly different from the value for vehicle treatment $(p<0.05)$.

(b) Relationships between the Mitochondrial Swelling and Release of Cytochrome c $(n=4)$

O, vehicle, $\diamond, 30 \mu \mathrm{M}$ MBM, $\square, 100 \mu \mathrm{M}$ MBM, $\triangle, 300 \mu \mathrm{M}$ MBM. Significant relationships between the mitochondrial swelling and the release of cytochrome $\mathrm{c}$ were seen.

determine the differences in the inhibitory effect between MCB and MBM. Therefore, we focused on the effect of MBM following studies. We next examined the effect of MBM on $\mathrm{Ca}^{2+}$ and $\mathrm{PhAsO}$-induced release of cytochrome $\mathrm{c}$ from the isolated brain mitochondria. MBM dose-dependently attenuated the release of cytochrome c. We also showed that there was the close relation between the release of cytochrome $\mathrm{c}$ and mitochondrial swelling as shown in Fig. 3b. Therefore, it is suggested that MBM may prevent the formation of thiol cross-linking of ANT, consequently inhibits mitochondrial swelling and the release of cytochrome $\mathrm{c}$ from the mitochondria, which is capable of inducing apoptotic pathways. As calcium accumulation in the cell and oxidative damage frequently occur under conditions of ischemia followed by reperfusion, we have hypothesized that MBM may have the ability to prevent neuronal cell damage through an inhibition of mitochondrial swelling and cytochrome $\mathrm{c}$ release. As shown in Fig. 4 and Table 1, in vivo treatment with 

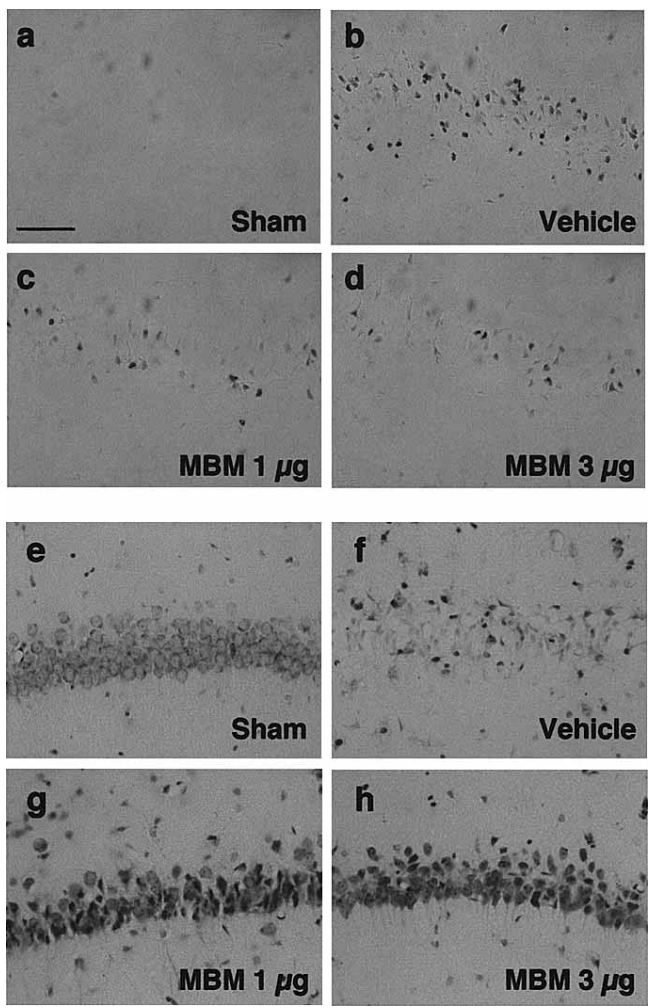

Fig. 4. Effects of MBM on the Number of TUNEL-Positive Cells (a-d) and Cresyl-Violet (Nissl)-Stained Cells $(\mathrm{e}-\mathrm{h})$ in the Hippocampal CA1 at $72 \mathrm{~h}$ after Transient Global Cerebral Ischemia

Representative high magnification views of the hippocampal CA1 region from sham (a, e), vehicle (b, f)-, and MBM [1 $\mu \mathrm{g}(\mathrm{c}, \mathrm{g})$ and $3 \mu \mathrm{g}(\mathrm{d}, \mathrm{h})]$-treated ischemic animals at $72 \mathrm{~h}$ after reperfusion. Bar $=50 \mu \mathrm{m}$.

Table 1. Effects of MBM on the Number of TUNEL-Positive Cells and Survival Neurons after Transient Global Cerebral Ischemia

\begin{tabular}{lcccc}
\hline \hline & Sham & $\mathrm{I} / \mathrm{R}$ & $\mathrm{I} / \mathrm{R}+1 \mu \mathrm{g}$ MBM & $\mathrm{I} / \mathrm{R}+3 \mu \mathrm{g} M B M$ \\
\hline TUNEL & 0 & $494.6 \pm 99.3$ & $245.7 \pm 29.2^{\#}$ & $143.6 \pm 49.0^{\#}$ \\
Nissl & $635.8 \pm 12.6$ & $50.5 \pm 32.7^{*}$ & $308.4 \pm 29.0^{\#}$ & $483.4 \pm 60.5^{\#}$
\end{tabular}

Values represent the mean numbers of cells \pm S.E.M. $/ \mathrm{mm}^{2}$ of the hippocampal CA1 regions from 4 (TUNEL staining) or 5 (Nissl staining) animals. * Significantly different from the sham-operated groups. \#Significantly different from the I/R groups. I/R indicates ischemia and reperfusion group.
MBM attenuated the increase in the number of TUNEL-positive cells and neuronal cell death in the hippocampal CA1 region after transient global cerebral ischemia. Although further studies will be required to clarify the precise mechanisms for in vivo effects of MBM on neuronal cell death, MBM may contribute to an inhibition of mitochondria-associated apoptotic pathways through an attenuation of the mitochondrial swelling and the release of cytochrome $\mathrm{c}$ from mitochondria.

\section{REFERENCES}

1) Zoratti M., Szabo I., Biochim. Biophys. Acta, 1241, 139-176 (1995).

2) Kristal B. S., Dubinsky J. M., J. Neurochem., 69, 524-538 (1997).

3) Susin S. A., Zamzami N., Kroemer G., Biochim. Biophys. Acta, 1366, $151-165$ (1998)

4) Friberg H., Connern C., Halestrap A. P., Wieloch T., J. Neurochem., 72, 2488-2497 (1999).

5) Berman S. B., Watkins S. C., Hastings T. G., Exp. Neurol., 164, 415425 (2000).

6) Andreyev A., Fiskum G., Cell Death Differ., 6, 825-832 (1999).

7) Haworth R. A., Hunter D. R., Arch. Biochem. Biophys., 195, 460-467 (1979).

8) Crompton M., Costi A., Biochem. J., 245, 915-918 (1987).

9) Gunter T. E., Pfeiffer D. R., Am. J. Physiol., 258, C755-786 (1990).

10) Halestrap A. P., Davidson A. M., Biochem. J., 268, 153-160 (1990).

11) Bernardi P., Scorrano L., Colonna R., Petronilli V., Di Lisa F., Eur. J. Biochem., 264, 687-701 (1999).

12) Pulsinelli W. A., Brierley J. B., Plum F., Ann. Neurol., 11, 491-498 (1982).

13) Lipton P., Physiol. Rev., 79, 1431-1568 (1999).

14) Petronilli V., Costantini P., Scorrano L., Colonna R., Passamonti S., Bernardi P., J. Biol. Chem., 269, 16638-16642 (1994).

15) Chernyak B. V., Bernardi P., Eur. J. Biochem., 239, 623-630 (1996).

16) Sims N. R., J. Neurochem., 55, 698-707 (1990).

17) Halestrap A. P., Biochem. J., 278, 715-719 (1991).

18) Sugio K., Horigome N., Sakaguchi T., Goto M., Stroke, 19, 922 (1988).

19) Takagi N., Sasakawa K., Besshoh S., Miyake-Takagi K., Takeo S., J. Neurochem., 84, 67-76 (2003).

20) Zamzami N., Kroemer G., Nat. Rev. Mol. Cell Biol., 2, 67-71 (2001).

21) Halestrap A. P., Woodfield K. Y., Connern C. P., J. Biol. Chem., 272, 3346-3354 (1997).

22) Packer M. A., Murphy M. P., Eur. J. Biochem., 234, 231-239 (1995).

23) Balakirev M. Y., Khramtsov V. V., Zimmer G., Eur. J. Biochem., 246, $710-718$ (1997) 